\title{
Prevention in perinatal and postpartum depression - non systematic review of high-quality evidence studies
}

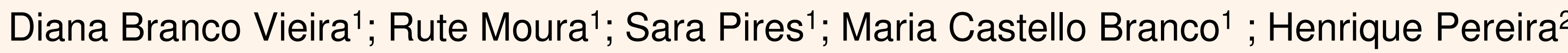 \\ 1- Medical resident in Child and Adolescent Psychiatry, Child and Adolescent Psychiatry Department, Hospital Dona Estefânia, Centro \\ Hospitalar Universitário de Lisboa Central, Portugal \\ 2- Child and Adolescent Psychiatrist, Child and Adolescent Psychiatry Department, Hospital Dona Estefânia, Centro Hospitalar \\ Universitário de Lisboa Central, Portugal
}

Objectives: To perform a qualitative non-systematic review of highquality evidence studies concerning prevention of perinatal/postpartum depression (PPD). The scope of this work excludes the treatment or measures to be taken in woman who meet diagnostic criteria.

Background: Depression is one of the most common complications of childbearing, affecting as many as 1 in 7 women. It impacts both the mother and child, with consistently demonstrated deleterious effects of PPD on cognitive and emotional development during infancy and later childhood. Given the public health relevance of PPD, preventing it has great potential for clinical efficacy.

Materials and methods: Bibliographic research of randomized controlled trials, systematic reviews and meta-analysis in the platforms PubMed, Elsevier, Science Direct, PsycINFO with the keywords "perinatal depression", "postpartum depression", "preventing", "prevention", "preventive interventions".

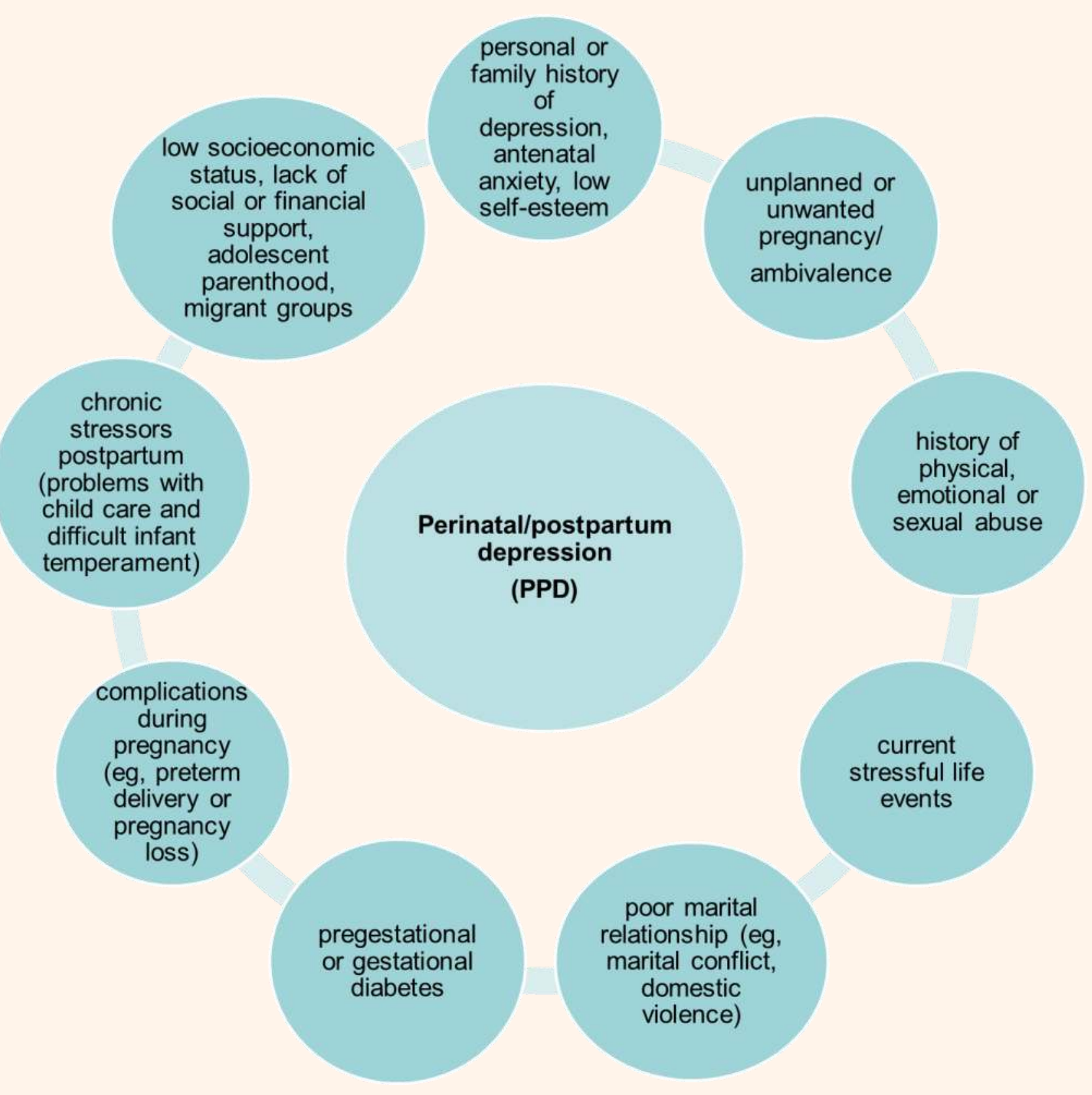

Figure 1. Psychological and social risk factors for PPD
Results: There are numerous known psychological and social risk factors for PPD - figure 1. Most studies use the Edinburgh Postnatal Depression Scale (EPDS) to ascertain a cut-off (EPDS score > 12 indicates PPD). However, there is no accurate screening tool for identifying women at risk and who might benefit from preventive interventions.

According with the US Preventive Services Task Force - USPSTF (1), to the present there is convincing evidence that counselling interventions, such as cognitive behavioural therapy (CBT) and interpersonal therapy (IPT), are effective in preventing PPD. These findings are supported by others systematic reviews, meta-analysis and randomized controlled trials.

In a review performed by the Cochrane (3.) the authors conclude that psychosocial and psychological interventions significantly reduce the number of women who develop PPD. They also state that interventions aiming 'at-risk' mothers may be more beneficial and practicable than those including the general population of mothers.

The research on family-based interventions is still in the early stages. There is limited or mixed evidence that other interventions such as physical activity, education, pharmacotherapy, dietary supplements are effective as preventive interventions.

A pragmatic approach is shown in figure 2 . The literature shows no data on the ideal timing for interventions, but continuing assessment of risks during pregnancy and the immediate postpartum would be rational, and referral could happen at any time.

Conclusions: There is evidence to refer women who are at increased risk of PPD to counselling interventions. Further research should address significant gaps in numerous areas relevant to this theme.

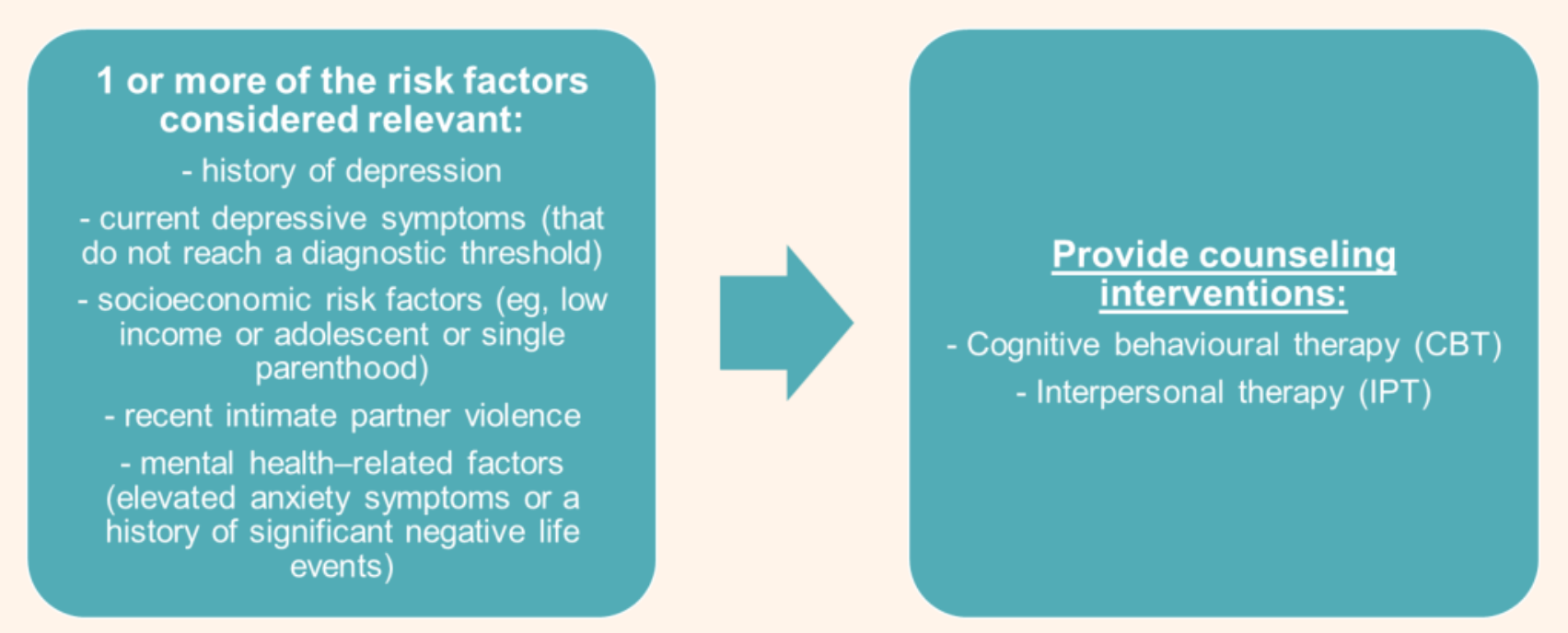

Figure 2. A pragmatic approach could be to provide counseling interventions to women with 1 or more of the risk factors considered relevant (adapted from Curry SJ, et al., USPSTF)

6. Sockol LE. A systematic review of the efficacy of cognitive behavioral therapy for treating and preventing perinatal

Cluxton-Keller $F$, Bruce $M L$. Clinical effectiveness of family therapeutic interventions in the prevention and treatment of perinal depr. Csion: A systematic review and meta-analysis. PLOS One 2018:13(6):00198730. Werner $E$ et al. Preventing postpartum depression: review and recommendations. Arch Womens Ment Health. $2015 \mathrm{Feb} ; 18(1): 41-60$.

Sparling TM, et al. The role of diet and nutritional supplementation in perinatal depression: a systematic review. Matern Child Nutr. 2017 Jan;13(1). 